\title{
Basophilic Hypertrophic Focus
}

National Cancer Institute

\section{Source}

National Cancer Institute. Basophilic Hypertrophic Focus. NCI Thesaurus. Code C139137.

A central point or locus characterized by hypertrophy and basophilia. 